\title{
O TRABALHO DE ENFERMAGEM NA ALTA DE CRIANÇAS HOSPITALIZADAS: articulação da atenção hospitalar e básica ${ }^{a}$
}

\author{
Raquel Vicentina Gomes de Oliveira SI LVA ${ }^{\text {b }}$ F lávia Regina Souza RA M OSc
}

\section{RESUM 0}

E sta pesquisa objetivou conhecer a atuação do enfer meiro no processo de alta hospital ar de crianças na articulação entre A tenção H ospitalar e Básica, na per spectiva da integ ral idade do cuidado. A proposta metodológica teve uma abordagem qual itativa-exploratória-descritiva, utilizando entrevistas semi-estruturadas com sete enfermeiros da atenção hospitalar e seis da atenção básica, col etadas entre dezembro de 2008 e janeiro de 2009, em seus locais de trabal ho. Constatou-se uma lacuna no processo de trabal ho de enfermagem no pós-alta hospitalar da criança, por praticamente não existir a articulação da atenção hospitalar e básica. A atuação do enfer meiro da atenção básica não acontece com freqüência, pois, dificilmente consegue identificar a criança em alta hospitalar. 0 agir integ ral de cada enfermeiro nem sempre consegue ultrapassar seu espaço de trabal ho, sendo que os profissionais envolvidos reconhecem que existem limitações em sua atuação nessa articulação, as quais remetem à necessidade de reorganização do seu processo de trabalho.

D escritores: Assistência integral a saúde. Enfermagem pediátrica. Papel do profissional de enfermagem. T rabalho.

\section{RESUMEN}

E sta investigación tuvo como objetivo conocer, desde la perspectiva de la integralidad del cuidado, la labor del enfermero en el proceso de alta hospitalaria del niño, en el articulación entre A tención H ospitalaria y la Básica. L a propuesta metodológica tuvo un abordaje cualitativo y exploratorio y descriptivo, utilizando entrevistas semiestructuradas con 7 enfer meros de la atención hospitalaria y 6 de la atención básica, recogidos entre diciembre de 2008 y enero de 2009, en sus propios lugares de trabajo. E I resultado mostró un vacío en el proceso del trabajo de enfermería en el post alta hospitalaria del niño, debido a que prácticamente no existe una articulación entre la atención hospitalaria y la básica. E n ese proceso, la labor del enfermero de la atención básica no acontece con frecuencia, por lo que difícil mente consigueidentificar al niño en el alta hospital aria. L a labor integral de cada enfer mero no si empreultrapasa su espacio de trabajo. L os propios profesionales reconocen que en esa articulación existen limitaciones en su labor que hacen necesaria la reorganización el proceso de trabajo.

Descriptores: A tención integral de salud. E nfermería pediátrica. R ol de la enfermera. T rabajo.

Título: E I trabajo de enfermería en el alta del niños hospitalizados: la articulación de la atención hospitalaria y la básica.

\section{ABST RACT}

This study aims to ascertain the nurse's role in the discharge process of children from hospitals, in the articulation betw een hospital careand primary care, from the point of view of comprehensivecare T hemethodology has a qualitative exploratory descriptiveapproach. It uses semi-structured inter views with seven nurses of hospital care and six of primary care, collected from D ecember 2008 to J anuary 2009, in their ow $n$ w orkplaces. R esults show a gap in the post-discharge nursing process, because ther eis virtually no articulation betw een hospital careand primary care P rimary health nurse often do not act in this process, because it is difficult to identify the child as discharged from a hospital. The actions of each nurse can not al ways go beyond their w orkplaces. P rofessionals involved in this process acknow ledgetheir limitations wherethis articulation is concerned, and this documents the necessity of a reorganization of the work process.

D escriptors: Comprehensive health care. P ediatric nursing. N urse's role. Work.

T itle: $\mathrm{N}$ ursing work in the discharge of hospitalized children: articulation betw een primary care and hospital care.

\footnotetext{
a Artigo originado da dissertação de M estrado apresentada em 2009 ao Programa de Pós-Graduação em Enfermagem da Universidade Federal de Santa Catarina (UFSC).

${ }^{b}$ M estre em Enfermagem, D outoranda pelo Programa de Pós-G raduação em Enfermagem da UF SC, Enfermeira A ssistencial da U nidade de Internação Pediátrica do H ospital U niversitário da U F SC, F lorianópolis, Santa Catarina, Brasil.

¿ D outora em E nfermagem, Professora A djunta do D epartamento e da Pós-G raduação em Enfermagem da UF SC, Pesquisadora e Bolsista de Produtividade em Pesquisa do CN Pq, Florianópolis, Santa Catarina, Brasil.
} 


\section{INT RODUÇÃO}

0 processo de trabalho em enfermagem é complementar e interdependente ao processo de trabaIho em saúde ${ }^{(1)}$.

A Enfermagem é uma prática social, cooperativa, com finalidade básica de atender às necessidades do indivíduo que, em seu percurso de vida, procura os serviços de saúde ${ }^{(2)}$. A enfermagem assiste ao indivíduo em sua integralidade como ser biológico e social ${ }^{(2)}$. 0 trabalho do enfermeiro pode ser desenvolvido em vários serviços de saúde, dentre eles encontram-se os hospitais e as U nidades L ocais de Saúde (U LS), sendo que, uma das parcelas de atuação da enfermagem ocorre durante a hospital ização e processo de al ta de crianças.

A hospitalização é traumática para a criança e "causa um estado de insegurança e ameaça psíquica"(3). N ela ocorre a interrupção da vivência com familiares e os procedimentos realizados pela $\mathrm{E} n$ fermagem causam, algumas vezes, danos físicos, emocionais e cognitivos aos envolvidos nesse processo. A criança hospitalizada e em processo de alta hospitalar é um ser fragilizado e necessita de atenção integral para promoção de sua saúde.

A pós a alta hospitalar é necessária a continuidade da assistência, quando a criança necessita de suporte do nível da atenção básica a saúde para uma melhor recuperação. A poiar esse processo da alta hospitalar corresponde a uma das dimensões da assistência do enfermeiro, seja do nível hospitaIar ou da atenção básica/ Equipe de E stratégia Saúde da Família (E SF ). É sua atribuição envolver-se na alta da criança e promover a continuidade da assistência do hospital ao domicílio, uma assistência integrada e comprometida. No entanto, esse processo para ser positivo, necessita de integração entre estes dois níveis de atenção.

Entretanto, a organização dos serviços ainda demonstra falta de comunicação entre os diversos níveis de atenção. 0 enfermeiro da U nidade Básica avalia como precário o funcionamento do sistema de referência e contra-referência ${ }^{(4)}$ e tal insuficiência é uma das principais dificuldades referidas pelos gestores municipais do sistema ${ }^{(5,6)}$.

Esta realidade, de inefetivo sistema de referência e contra-referência, dificulta o processo de articulação entre a unidade hospitalar e básica, bem como a integralidade do cuidado. A falta de informação e as limitações organizacionais do processo de trabal ho favorecem uma ruptura na assistência prestada.
N esta perspectiva, o hospital contribuiria para a integ ralidade quando o "sistema de saúde" fosse refer ência em determinadas situações e, após realizar 0 atendimento, fizesse uma adequada contrareferência, sendo a alta hospitalar consider ada um "momento privilegiado" para se trabalhar a integralidade ${ }^{(7)}$.

Por outro lado, a ESF poderia facilitar a construção de um sistema integrado à medida que vaIoriza os atributos necessários para uma atenção básica resolutiva, especialmente pelo princípio da integralidade e pelo acesso da população a todos os níveis de atenção à saúde ${ }^{(8)}$.

$\mathrm{N}$ a L ei Orgânica da Saúde 8.080/90, a construção do Sistema Ú nico de Saúde (SU S) é norteada por alguns princípios e diretrizes ${ }^{(9)}$, dentre os quais a integralidade. Esta, deve ser percebida em uma dimensão individual, relacionada à intensidade do cuidado no ato de atenção à saúde e em uma dimensão sistêmica, em relação a uma atenção contínua, através de ações articuladas em rede que promovam a coordenação do cuidado entre os diversos serviços de saúde(8).

Como um elemento que favorece a integralidade da assistência e promove saúde, percebe-se a importância da assistência de enfer magem, por sua presença continuada junto à criança hospitalizada, e ressalta-se o seguimento pós-alta hospitalar como parte deste cuidado.

E ste artigo relata parte dos resultados de uma dissertação(10). T em como objetivo conhecer a atuação do enfermeiro no processo de alta hospitalar de crianças na articulação entre A tenção H ospitaIar e Básica, na perspectiva da integ ralidade do cuidado.

\section{MATERIAIS E MÉTODOS}

Este estudo teve como proposta metodológica a abordagem qualitativa(11) de caráter exploratório-descritivo. Foi desenvolvido em um hospital universitário público, e em U nidades Locais de Saúde (U LS) de um M unicípio da região sul do Brasil.

Os participantes do estudo foram 13 enfermeiros/as: sete atuantes em uma U nidade de Inter nação Pediátrica (UIP) e seis atuantes na atenção básica/ ESF, selecionados através de um sorteio prévio, realizado pela pesquisadora. $\mathrm{Na}$ Atenção Básica, com o propósito de alcançarmos um resultado que correspondesse à totalidade deste 
M unicípio, realizamos um sorteio em cada uma das cinco Regionais de Saúde. Salientamos que uma enfermeira da atenção básica, por ser coordenadora da Saúde da Criança do município foi intencionalmente selecionada, por poder contribuir no enriquecimento da discussão.

A delimitação do número de entrevistas baseou-se no critério de "saturação" qual itativa ${ }^{(12)}$ e foi considerado como critério de inclusão, o tempo mínimo de um ano de atuação desses enfermeiros em suas áreas específicas e que demonstraram aceitação voluntária em participar da entrevista.

$\mathrm{Na}$ coleta de dados foi utilizada a entrevista semi-estruturada, pautada por um roteiro previamente elaborado(13), realizadas individualmente pela pesquisadora, no ambiente de trabalho dos participantes, em data e horários combinados, no período de dezembro de 2008 a janeiro de 2009, registradas por gravação de áudio e, posteriormente, transcritas na íntegra. A pós serem digitadas, foi realizada leitura e releitura e análise individual das entrevistas; sel eção e identificação das unidades de significado; organização das unidades de significado por semel hança de conteúdo e; constituição das categorias ${ }^{(14)}$.

$\mathrm{Na}$ análise, utilizou-se como fer ramenta tecnológica o software AT LAS. T i. Seu uso favorece a estocagem dos dados e sua análise ${ }^{(15)}$. E ntretanto, a codificação é produto do pensamento e da versatilidade do pesquisador ${ }^{(16)}$.

A pós a categorização, foi realizada uma análise e discussão dos dados, sob a ótica do referencial teórico: processo de trabalho de enfermagem e integral idade do cuidado.

As fases desta pesquisa foram fundamentadas na Resolução 196/ 96 do Consel ho N acional de Saúde ${ }^{(17)}$. Este projeto foi aprovado pelo Comitê de Ética e Pesquisa com Seres H umanos da U niversidade Federal de Santa Catarina (certificado 291). 0 anonimato foi garantido com o uso de códigos para identificar suas falas (E IESF, E IUIP), com a letra "E", seguida do número e do local de trabalho - Estratégia Saúde da Família (ESF) e U nidade de Internação Pediátrica (UIP). A autorização foi obtida através do Termo de Consentimento Livre e E sclarecido.

\section{RESULTADOSE DISCUSSÃO}

A alta hospitalar é entendida pelos entrevistados como um processo que inicia com a interna- ção, inclui o período que antecede a saída da criança do hospital e o período após a saída desta do hospital, incluindo o seu seguimento pós-alta na comunidade onde reside.

Neste processo, existe um agir multiprofissional, sendo que dentre estes encontra-se 0 enfermeiro, com seu processo de trabalho e sua assistência à saúde da criança.

0 dia da al ta é importante tanto para a criança, como para a equipe de enfermagem. Para o primeiro, gera alegria e insegurança e, para o segundo, um momento de responsabilidade e segurança em relação às orientações que deverão ser realizadas $^{(18)}$.

0 assistir à criança em processo de alta hospitalar está inserido como parte do cuidado do enfermeiro da atenção hospitalar e básica, como um elemento que favorece a integralidade da assistência e promoção da saúde.

Por sua vez, percebemos a integralidade "como um conjunto articulado e contínuo das ações e ser viços preventivos e curativos, individuais e coletivos, exigidos para cada caso em todos os níveis de complexidade do sistema"(9).

\section{A atuação no processo de alta hospitalar: o enfermeiro da atenção hospitalar}

Tomando por base os relatos dos sujeitos de pesquisa acerca do processo de trabal ho de enfermagem à criança em processo de alta realizado na atenção hospitalar, pode-se afirmar que, na maioria das vezes, é realizada uma prática de cuidado integral, porém em uma dimensão individual ${ }^{(8)}$, ou seja, enquanto este binômio (criança/ família) encontra-se internado, existe um esforço por parte da equipe para assistir todas as suas necessidades.

As enfermeiras da UIP conseguem atuar de forma direta, durante 0 processo de internação e na alta propriamente dita.

A gente consegue trabalhar com este paciente durante o processo de inter nação [ .... . . M inha atuação édireta, [ ...] e principalmenteno final [ ...] eu procuro [ ...] resgatar, trabalhar aquilo que foi perdido com a doença (E1 UIP).

$\mathrm{Na}$ chegada da criança e sua família à UIP, é realizado um acolhimento e um histórico de enfermagem. Através deste, são realizadas prescrições de enfermagem individuais, sendo que estas são 
trabal hadas durante o período de internação e alta hospitalar, na qual são resgatadas as necessidades da criança.

$\mathrm{N}$ a alta eu resgato 0 histórico pra ver se todas as necessidades da criança [ ...] foram trabalhadas. [ ...] (E 1 UIP).

$\mathrm{Na}$ alta hospitalar, as enfermeiras procuram reorientar o responsável pela criança sobre informações recebidas durante a internação e na própria alta, inclusive as orientações recomendadas pelo médico, e quando necessário, é fornecido a medicação para continuidade ao tratamento.

Essa questão da orientação apareceu na maioria das falas dos entrevistados, quando questionados acerca de seu processo de trabal ho, seja durante a internação ou na alta hospitalar.

[ ...] orientar as necessidades dessa família, tanto no contexto intra-hospitalar como extra-hospitalar, como ela deve proceder nessa alta (E 3 UIP).

N esse caso, a orientação pode ser vista como um instrumento que contribui para a integralidade do cuidado, ainda que voltada para uma dimensão individual, sendo utilizada para garantir a continuidade do cuidado. Considera-se que as orientações devem ser real izadas durante toda a internação, e reforçados os pontos mais importantes no momento da alta(18).

Segundo as enfermeiras da UIP, na alta hospitalar, quando ocorre o encaminhamento direto para a atenção básica, na maioria das vezes isso ocorre informalmente, de forma verbal. $\mathrm{N}$ ão existe uma prática instituída, um protocolo para orientar este agir.

Observa-se que somente em alguns casos muito específicos ocorre articulação do processo de trabalho de enfermagem entre atenção hospitalar e básica por escrito.

I sso a gente costuma fazer é com os indígenas, porque existe essa ligação [ ...] (E 6 UIP).

As falas seguintes apontam que os profissionais reconhecem que existem limitações em sua atuação na articulação entre atenção hospitalar e básica, no que se refere ao processo de alta hospitalar, bem como, uma lacuna na assistência, que necessita ser preenchida e reorganizada.
[ ...] a gente não atua como deveria [ ...] damos mais orientações (E 4 UIP).

Sempre que a criança [ ...] recebe alta, eu me preocupo em conversar [ ...]. . M as eu tenho consciência de que é bastante limitada a minha atuação [ ...] deveria existir um processo de alta que deveria englobar todos esses cuidados [ ...] como fazer um contato com a unidade de saúde [ ...] pra estabelecer uma continuidade (E 6 UIP).

$\mathrm{Na}$ perspectiva da integralidade em saúde, em uma "dimensão sistêmica"(8), acredita-se que no processo de al ta hospitalar existe uma necessidade que vai além das orientações. A pesar de se reconhecer que esta é um importante instrumento da assistência, muitas vezes não é 0 suficiente para impedir a reinter nação da criança, haja vista que, geralmente, essas orientações e informações não chegam às ULS. Algumas vezes a informação chega muito tempo após a alta, dificultando assim a atuação da equipe da ESF. Somente um enfermeiro da atenção básica relatou que a criança foi a ULS após receber alta hospitalar, bem como levou as orientações dos profissionais da atenção hospitalar.

I dentifica-se também que, a enfermeira da UIP encaminha a criança para 0 assistente social. E ste, juntamente com outros profissionais, participa do "intercâmbio": um momento multidisciplinar, onde diversos profissionais reúnem-se e discutem os casos das crianças internadas, determinando assim, a articulação dos diver sos saberes.

Quando os diferentes profissionais realizam troca de informações, conhecimentos acerca do trabal ho, estudos de casos e decidem em equipe uma resolução para os problemas de saúde dos pacientes, aí acontece a articulação das ações ${ }^{(19)}$.

[ ...] a genteacaba sabendo da situação de todos os ângulos dessa família [ ...] criança [ ...] (EG UIP).

A ssim, o "intercâmbio" é uma prática realizada no processo de trabalho de enfermagem da atenção hospitalar, na qual, reflete a integralidade do cuidado. A pesar disso, nem todos os profissionais lembraram-se de citá-lo. I sso leva-nos a pensar que, apesar desse princípio ser praticado no cotidiano da enfermagem, de estar presente no agir desses profissionais, não está presente no falar.

O utro aspecto identificado é a posição importante que o enfermeiro ocupa no processo de alta hospitalar: "peça especial", "visão ampliada" (E6 ESF). 
Por fim, os sujeitos trabalhadores de enfermagem da atenção hospitalar reconhecem que a alta hospitalar da criança rompe o cuidado integral executado durante a internação.

M as a partir do momento que a criança ganha alta, eu acho que quebra esse vínculo de integralidade do cuidado (E 2 UIP).

A pesar de a integralidade poder estar presente no cuidado individual diário do enfermeiro, membro de uma equipe, percebe-se que no momento da alta hospitalar, esta não consegue alcançar sua intensidade em potencial.

\section{A atuação no processo de alta hospitalar: o enfermeiro da atenção básica}

N o processo de trabalho na atenção básica, os enfermeiros colocam que sentem dificuldade em praticar um atendimento integral a saúde da criança, principalmente no processo de alta hospitalar. U ma das dificuldades citadas foi a presença do especialista pediatra na ULS que, muitas vezes faz 0 atendimento a todas as crianças da área de abrangência.

[ ...] a gente não tem contato com as crianças, por essa presença do especialista dentro da unidade, a gente não trabalha como equipe de saúde da família no atendimento integral a criança [ ...] 0 cuidado dire tamente a gente tem como se fosse um buraco [ ...] 0 especialista [ ...] acaba atendendo todas as crianças (E3 ESF).

E videncia-se que nem todos os enfermeiros da atenção básica conseguem realizar consultas à criança, sendo que poucos intercalam suas consultas com o médico de saúde da família conforme preconiza o programa do município. Nessas consultas, al gumas vezes, o enfermeiro identifica crianças em processo de alta hospitalar e procura dar continuidade no cuidado.

O utras vezes, os enfermeiros da atenção básica ficam sabendo da alta hospitalar da criança, através do A gente Comunitário de Saúde (ACS) e ou através dos familiares das crianças que recebem orientação das enfermeiras da atenção hospitalar para procurarem a ULS.

E ssa contra- refer ência [ ...] passa a acontecer quando algum profissional de saúde, ou ACS identifica que es- sa criança tevealta hospitalar [ ...] ou a própria família vem nos avisar [ ...] O u se essa unidade funciona muito bem, e se essa criança for monitorada pelo ACS a gente vai saber a tempo. [ ...] ainda vejo que não é comum no nosso processo de trabalho na unidade de saúde (E 6 ESF).

A pesar disso, geralmente 0 enfermeiro não consegue atuar nesse processo, pois, na maioria das vezes, não fica a par da internação da criança, ou quando é notificado já ocorreu a alta hospitalar há dias. Essas situações fragmentam a assistência integral, o processo de trabalho de enfer magem não contribui para a integralidade, o que reflete diretamente na promoção de saúde da criança. Ressalta-se a importância do ACS e de seu agir, em que acompanhar a criança é uma de suas atribuições, bem como repassar as informações a sua equipe. 0 não atuar da equipe de ESF no pós-alta da criança pode resultar em uma nova reinter nação.

A pós a identificação da criança em processo de alta hospitalar, os enfer meiros procuram obter informações acerca da internação com o responsável pela criança e, também, as informações encaminhadas pelos profissionais da atenção hospitalar.

I dentifica-se que poucas crianças possuem registradas em suas cader netas, anotações relacionadas à sua inter nação hospitalar, outras trazem somente o cartão de alta com o diagnóstico e receituário médico. São raras as situações nas quais ocorrem todos os registros acerca da internação e prescrições ou indicações para continuidade do tratamento na atenção básica, o que facilitaria a integralidade do cuidado.

Além das consultas nas U LS, outra atividade realizada pelo enfermeiro é a visita domiciliar (VD) à criança no processo de alta hospitalar. A VD é uma das atribuições do enfermeiro e muitas vezes, é a única oportunidade, que este encontra para realizar consulta a criança.

Por outro lado, nas falas abaixo, alguns dos enfermeiros reconhecem que nem sempre conseguem realizar VD, dando prioridade, aos casos mais críticos.

Que pelo menos eu priorize as situações de riscos [ ...] às vez es é uma demanda muito grande, você não consegue dar conta da criança mais saudável, mas muito menos vocêda conta daquela queéde risco (E 6 ESF).

E m uma pesquisa acerca da visão da enfermeira sobre as articulações das ações de saúde en- 
tre profissionais de equipes de saúde da família, 0 excesso de demanda de usuários pelo serviço, assim como nesta pesquisa, também aparece como dificuldade, gerando falta de tempo para planejamento e articulação das ações em saúde ${ }^{(19)}$.

No processo de trabalho de enfermagem, existe uma preocupação do enfermeiro em agir integralmente, quando repassa as informações resgatadas durante a VD para a equipe ESF e principalmente para o pediatra.

$\mathrm{N}$ a fala seguinte, é referida outra maneira do enfer meiro identificar a criança em processo de alta hospitalar, durante 0 acol himento. E ste passa a ser utilizado como uma ferramenta, para que, tanto os profissionais, como os serviços de saúde prestem uma assistência integral, um alicerce da integralidade em saúde ${ }^{(10)}$.

Os enfermeiros mencionaram que quando conseguem identificar as crianças em processo de alta hospitalar durante 0 acolhimento, procuram realizar VD para dar continuidade no atendimento.

Tá identificando no acolhimento [ ...] história de internação hospitalar [ ...] pra posterior visita ou acompanhamento do ACS [ ...] (EI ESF).

Observamos que, apesar da importância do enfer meiro realizar a VD, em algumas situações quem realiza é somente o ACS, que serve de elo entre a criança e o profissional de saúde. Este traz informações ao enfermeiro supervisor sobre a criança e também procura trazê-las para consulta com a equipe $E S F$.

N esta outra fala, desvela-se a falta de informação dos enfermeiros e sua equipe acerca de inter nações, reinternações e óbitos de suas crianças.

[ ...] algumas crianças que morrem após 28 dias, são crianças quejá estão em situação de risco, re- hospitalizada ecuja equipenem sabia dessa existência [ ...] (E 6 ESF ).

E ssa situação confirma a lacuna existente no processo de trabalho de enfermagem no pós-alta da criança hospitalizada e denota a importância de investimento na organização do sistema de referência e contra-referência mais efetivo. Revela também o reconhecimento desse fato pelos gestores e sua preocupação em rever a atual prática realizada.

\section{CONSIDERAÇÕES FINAIS}

0 enfermeiro da atenção hospitalar consegue, na maioria das vezes, atuar na perspectiva da integralidade durante a permanência da criança no hospital. Entretanto, no seguimento pós-alta, identifica-se uma fragilidade no cuidado integral por praticamente não existir uma articulação da atenção hospitalar e básica nesse processo. Os próprios profissionais reconhecem que existem limitações em suas atuações nessa articulação, bem como, uma lacuna na assistência, que necessita ser preenchida por uma reorganização do processo de trabal ho.

A atuação do enfermeiro da atenção básica nesse processo não acontece com freqüência, pois nem sempre consegue identificar a criança em processo de al ta hospitalar. A pós a identificação, o enfermeiro encontra dificuldades para dar continuidade à atenção integral à criança, seja por não atuar em consultas às crianças, não conseguir realizar visitas e ou identificar a criança somente muito tempo após a alta.

Assim, constata-se uma lacuna no processo de trabal ho de enfermagem na alta, pós-alta da criança e na articulação da atenção hospitalar e básica. Percebe-se um conflito quando a integralidade do cuidado é vista como idéia de totalidade e o processo de alta hospitalar como algo que ultrapassa as paredes institucionais, entretanto a atuação do enfermeiro não consegue al cançar a integralidade "sistêmica"(8). 0 agir integral de cada enfermeiro não ultrapassa seu próprio espaço de trabalho, estabelecendo-se aí um vazio na assistência.

A articulação do trabalho do enfermeiro da atenção hospitalar e básica/ ESF no processo alta hospitalar da criança favoreceria a integralidade do cuidado. U m cuidado integral requer uma assistência que rompa os limites da instituição hospitalar em encontro à equipes de ESF e a elaboração de um caminho que contribua para o fortalecimento da referência e contra-referência deste processo.

Diante do exposto, espera-se que o estudo tenha incitado questionamentos e que estimule 0 desenvolvimento de novos estudos a cerca da temática e de novas práticas. Consideramos que 0 estudo contribuiu para uma reflexão das práticas executadas e nas futuras a serem desenvolvidas na Enfermagem Pediátrica. Está diretamente relacionado com os demais artigos desta revista, ao procurar compartilhar e agregar conhecimentos, no 
sentido de propiciar um cuidado qualificado e integral, tendo em vista, a escassez de estudos nesta temática.

\section{REFERÊ NCIAS}

1 Capella BB. U ma abordagem sócio-humanista para um "modo de fazer" o trabalho de enfermagem [ tese] . F lorianópolis: U niver sidade F ederal de Santa Catarina; 1996.

2 Pires DA. Estrutura objetiva do trabalho em saúde. In: L eopardi M T, organizadora. Processo de trabalho em saúde: organização e subjetividade. F Iorianópolis: Papa-Livros; 1999. p. 25-48.

3 L aurent M CR, D ias VL M . A sistematização da assistência de enfermagem em pediatria. Porto Alegre: Artmed; 2006. (Programa de A tualização em E nfermagem: saúde da criança e do adolescente; ciclo 1, módulo 1).

4 Juliani CM , Ciampone M H T. Organização do sistema de referência e contra-referência no contexto do Sistema Ú nico de Saúde: a percepção de enfer meiros. Rev Esc Enferm U SP. 1999;33(4):323-33.

5 M inistério da Saúde (BR ). Avaliação da implementação do Programa de Saúde da Família em dez grandes centros urbanos. Brasília (DF); 2002.

6 Rocha PM , U choa AC, Rocha N SPD, Souza E CF, Rocha M L, Pinheiro T X A. Avaliação do Programa Saúde da F amília em municípios do N ordeste brasileiro: velhos e novos desafios. Cad Saúde Pública. 2008;24 (Supl 1):S69-78.

7 Cecílio LCO, M erhy EE. A integralidade do cuidado como eixo da gestão hospital ar. In: Pinheiro R, M attos RA, organizadores. Construção da Integralidade: cotidiano, saberes e práticas em saúde. 3a ed. R io de Janeiro: I M S/ U ERJ/ A brasco; 2005.

8 Silva VC. 0 processo de implantação do sistema integrado de serviços de saúde em Vitória - ES: contribuição a discussão da integralidade na atenção à saúde [ dissertação] . Rio de Janeiro: Escola $\mathrm{N}$ acional de Saúde Pública; 2004.

9 M inistério da Saúde (BR). L ei no 8.080, de 19 de setembro de 1990: dispõe sobre as condições para a pro-

\section{Endereço da autora / Dirección del autor / Author's address:}

Raquel Vicentina G omes de Oliveira da Silva

R. Elpídio da Rocha, 345, Rio T avares

88048-398, Florianópolis, SC

E-mail:raquelenfer@yahoo.com.br moção, proteção e recuperação da saúde, a organização e o funcionamento dos serviços correspondentes e dá outras providências. Brasília (DF); 1990.

10 Silva RVG 0. 0 trabal ho de enfermagem na articulação entre atenção hospital ar e atenção básica no pós alta da criança: subsídios para a Integralidade [ dissertação] . F lorianópol is: U niversidadeF ederal de Santa Catarina; 2009.

11 M inayo M CS. Pesquisa social : teoria, método e criatividade. 21å ed. Petrópolis: Vozes; 2001.

12 M inayo M CS. 0 desafio do conhecimento: pesquisa qualitativa em saúde. 3ª ed. São Paulo: H ucitec; 1999.

13 M inayo M CS, Deslandes SF, G omes R. Pesquisa social: teoria, método e criatividade. 26a ed. Petrópolis: Vozes; 2007.

14 Dyniewicz A M . M etodologia da pesquisa em saúde para iniciantes. São Caetano do Sul: D ifusão; 2007.

15 Klippel M , Koetz AL, L acerda D P, T eixeira AR, A ntunes J J JAV. A pesquisa em administração com o uso de softwares QDA (Qualitative Data A nalysis). In: Anais do Simpósio sobre Excelência em G estão e Tecnologia; 2004 out 27-29; Rezende, Brasil. Rezende: A ssociação E ducacional D om B osco; 2004. p. $1-15$.

16 M oreira DA . 0 uso de prog ramas de computador na análise qual itativa: oportunidades, vantagens e desvantagens. Rev N eg. 2007;12(2):56-68.

17 M inistério da Saúde (BR), Consel ho N acional de Saúde. Resolução 196, de 10 de outubro de 1996: diretrizes e normas regulamentadoras de pesquisa envolvendo seres humanos. Brasília (D F ); 1996.

18 L acerda M R, Barbosa R, Z avadil ET C. Cuidados de enfermagem a crianças e a adolescentes em transplante de células-tronco hematopoiéticas. Por to A legre: A rtmed; 2008. (Programa de A tualização em E nfer magem: saúde da criança e do adolescente; ciclo 3, módulo 2).

19 Colomé ICS, Lima M ADS, D avis R. Visão de enfermeir as sobre as articulações das ações de saúde entre profissionais de equipes de saúde da família. Rev E sc Enferm USP. 2008:42(2):256-61.

Recebido em: 07/ 11/ 2010

A provado em: 16/ 05/ 2011 